\title{
Analysis of Microdisk/Microring's Surface Roughness Effect by Orthogonal Decomposition
}

\author{
Chengle Sui ${ }^{1}$, Qiangmin Wang ${ }^{2}$, Shilin Xiao ${ }^{1}$, Pingqing $\mathrm{Li}^{1}$ \\ ${ }^{1}$ State Key Lab of Advanced Optical Communication System and Networks, Shanghai Jiao Tong University, Shanghai, China \\ ${ }^{2}$ School of Information Security Engineering Shanghai Jiao Tong University, Shanghai, China \\ Email: sui_chengle@sjtu.edu.cn, qmwang@sjtu.edu.cn
}

Received 2013

\begin{abstract}
Application of micro-resonator is limited by different types of surface inhomogeneity. The 1-th derivative of inhomogeneity (i.e. $\left.\Delta r^{\prime}(\varphi)\right)$ affects the wave transport as well as the height of inhomogeneity (i.e. $\Delta r(\varphi)$ ). A method based on orthogonal decomposition is proposed to analysis both scattering mechanism respectively. Then surface roughness effect on Q-factor of micro-disk waveguide gallery mode (WGM) resonator is investigated with our method and the analysis fits well with FDTD simulation results.
\end{abstract}

Keywords: Orthogonal Decomposition; Micro-resonator; Surface Roughness Effect

\section{Introduction}

Micro-ring/micro-disk resonators are very elemental building blocks of integrated photonic device for its compact size and especially for its high quality factor (Q-factor) [1]. High-Q micro resonators are more and more widely applied in lasers, modulators and bio-sensors [2-4]. However, the Quality factor of the high-index-contrast system is still mainly constrained by scattering loss and absorption loss due to surface roughness [5].

Surface roughness effect on Q-factor and mode splitting are widely investigated in [5-9]. According to [10], wave transport along the waveguide can be affected by two scattering mechanisms: one is induced by the height of inhomogeneity (i.e. $\Delta r(\varphi))$, the other is due to the first-order derivative of inhomogeneity (i.e. $\Delta r^{\prime}(\varphi)$ ). In related works, the surface roughness is modeled as a random variable, and the scattering loss is calculated stochastically. But all of these methods either fail to observe the effect of or are too complex for calculation, because they cannot analyze independently the scattering loss due to both scattering mechanism. Therefore, if a simulation is needed to conduct for analysis, we cannot calculate the result accurately. For example, in [9], several Nano scale circular holes are carved evenly along the microdisk to make intentional surface roughness. In [6], random distributed inhomogeneity is approached by a number of randomly spaced rectangle asperities. All these approximation method is not very accurate, and cannot help us to further our understanding on the underlying scattering mechanism.
In this paper, a method based on orthogonal decomposition is proposed to simplify the calculation of surface roughness effect without losing accuracy. We try to approach the inhomogeneity infinitely by a linear combination of orthogonal basis we proposed, and then we can analyze the effect of $\Delta r(\varphi)$ and $\Delta r^{\prime}(\varphi)$ independently by carefully choosing proper orthogonal basis.

The paper is organized in the following way: in Section 2, the model of orthogonal basis is introduced, and then its correctness is proved by Fourier Transform. At the end of Section II, the utilization of the proposed orthogonal basis is studied in detail. In Section 3, we analyze the surface roughness effect on Q-factor of microdisk resonator at first. Then we conduct the analysis and FDTD simulation based on our method, the result of which validates our derivation.

\section{Model}

\subsection{Notations}

The side boundary of micro resonator is rough, and is shaped by closed contour $\mathrm{S}$ along the basis parallel to $\mathrm{z}$ basis, as depicted in Figure 1. The distance from center point to each side boundary is given by

$$
r(\varphi)=R+\Delta r(\varphi), \quad \varphi \in[-\pi, \pi]
$$

where $\mathrm{R}$ is the average radius and is a random variable with zero mean. The correlation function of is often fitted to Gauss distribution, i.e.

$$
\left\langle\Delta r\left(\varphi_{1}\right) \Delta r^{*}\left(\varphi_{2}\right)\right\rangle=\sigma^{2} \exp \left[-\left(\varphi_{1}-\varphi_{2}\right)^{2} / L_{c}^{2}\right]
$$


where Lc is the correlation length and $\sigma$ is the root mean square (rms) value of surface deviations. In addition, we have

$\langle\bullet \quad$ expectation operator

- 2-norm

$F(\bullet)$ elementary function

$\left[f_{k}(x)\right]^{i}$ i-th square of $f_{k}(x)$

$f_{k}^{i}(x)$ i-th order derivative of $f_{k}(x)$

Figure 2 shows one period of a sequence of orthogonal basis $\left\{f_{i}(x)\right\}$, which is orthogonal with each other, i.e.

$$
\begin{aligned}
\int_{-\infty}^{\infty} f_{i}(x) f_{j}(x) d x & = \begin{cases}1 & i=j \\
0 & i \neq j\end{cases} \\
\int_{-\infty}^{\infty} f_{i}^{\prime}(x) f_{j}^{\prime}(x) d x & =\int_{-\infty}^{\infty} f_{i}(x) f_{j}^{\prime}(x) d x \\
& = \begin{cases}1 & i=j \\
0 & i \neq j\end{cases}
\end{aligned}
$$

Finally, we define the period of orthogonal basis as L.

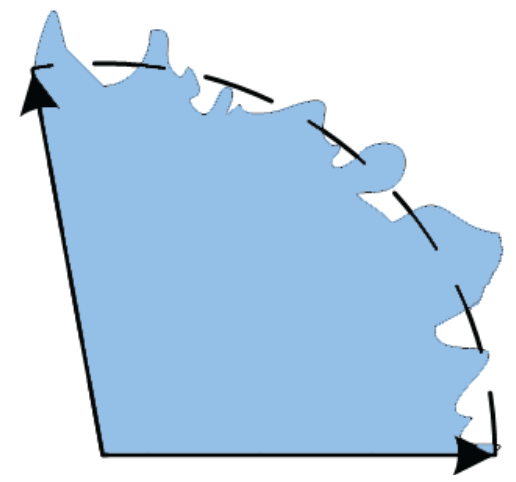

Figure 1. SNR degradation as function of laser line width.

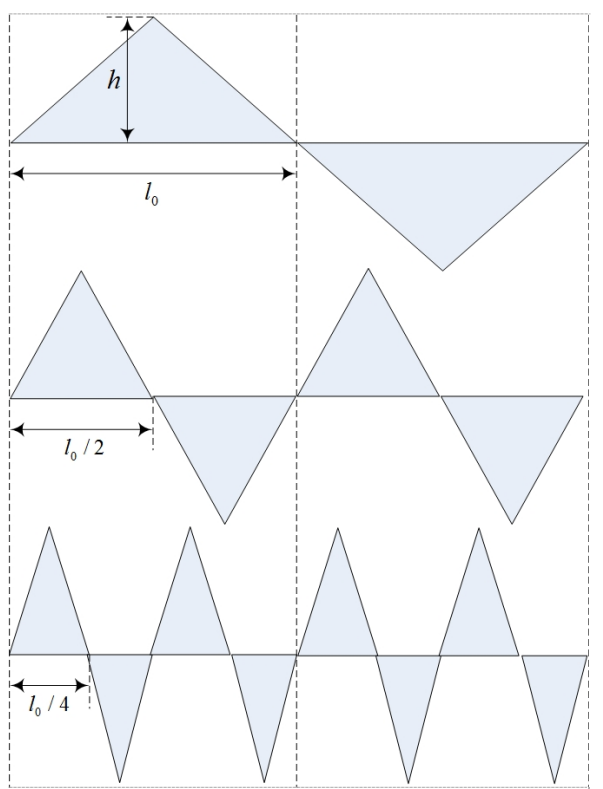

Figure 2. Schematic of orthogonal basis in one period.

\subsection{Orthogonal Decomposition}

Now we'll prove the fact by Fourier Transform that side wall inhomogeneity can be approached by orthogonal basis $\left\{f_{i}(\varphi)\right\}$ through orthogonal decomposition. The Fourier Transform of $f_{k}(x)$ is given by

$$
\begin{aligned}
& f_{k}(x)=\sum_{i=0}^{2^{k}-1} \sum_{n=-\infty}^{\infty}(-1)^{i} \mathrm{H}_{k n} \exp \left[j 2 \pi\left(n x+i l_{0}\right) / L\right] \\
& \mathrm{H}_{k n}=h \cdot \frac{\sin ^{2}\left(n \pi l_{0} / L\right)}{(n \pi / L)^{2}}
\end{aligned}
$$

According to [12-13], there would always be a linear combination of $\left\{f_{i}(\varphi)\right\}$ that can approach $\Delta r(\varphi)$ infinitely by meeting the condition

$$
\lim _{n \rightarrow \infty}\left\|\sum_{k=1}^{n} A_{k} f_{k}(\phi)-r(\phi)\right\|=0
$$

Therefore, if the orthogonal basis and its weight are carefully chosen, the orthogonal basis we proposed can accurately approximate the transmittance of micro resonator with rough surface.

For a specific resonator, several modes may be propagated along the waveguide. As it is stated in [5],

$$
\begin{aligned}
& \Delta r(\phi)=\frac{1}{2 \pi} \sum_{k} a_{k} \exp (j \cdot k \phi) \\
& a_{k}=\int_{0}^{2 \pi} \Delta r(\phi) \exp (-j \cdot n \phi) d \phi \\
& \left\langle a_{k} a_{k}^{*}\right\rangle=\frac{2 \pi^{3 / 2}\left(\sigma^{2} L_{c}\right)}{R} \exp \left[-\left(\frac{k L_{c}}{2 R}\right)^{2}\right]
\end{aligned}
$$

Reference [5] shows that each micro resonator is matched with a sequence of ak, and each ak corresponds to the mode splitting for resonant mode with azimuthal order $k$.

Now the way of getting the parameter of orthogonal basis we proposed will be revealed. Comparing (5) with (7), if we simply look into one specific mode splitting with azimuthal order $\mathrm{k}$, the surface roughness effect can be accurately approached by one specific orthogonal basis $f_{k}(\varphi)$ if only

$$
\begin{gathered}
2 \pi \cdot 2 \pi R / L=\phi+2 p \pi \quad p \in N \\
\left|h \cdot \frac{\sin ^{2}\left(n \pi l_{0} / L\right)}{(n \pi / L)^{2}} \sum_{i=0}^{2^{k}-1}(-1)^{i} \exp \left(j 2 \pi i l_{0} / L\right)\right| \\
=\mathrm{I}_{0} \cdot \sqrt{\frac{2 \pi^{3 / 2}\left(\sigma^{2} L_{c}\right)}{R} \exp \left[-\left(\frac{n L_{c}}{2 R}\right)^{2}\right]}
\end{gathered}
$$

Here, $I_{0}$ is normalized random variable whose mean value is 1 . If $L \ll 2 \pi R$, it's reasonable to assume that orthogonal basis spreads over micro resonator to infinite period. From (8), we can get that if the azimuth $\phi$ 
doesn't equal to 0 , the value of $2 \pi R / L$ won't be an integer, which means we cannot evenly distribute every period of orthogonal basis around the circle. But if $\mathrm{L}$ is chosen to be a very large number, the approximate error can be neglected. According (9), if L is given, we can get a simple relationship between $h$ and $l_{0}$.

Phase shift due to rough surface should also be taken into consideration when we investigate the mode splitting in micro-disk. The phase shift is uniformly distributed in the interval $[0,2 \pi)$, so the orthogonal basis can be modified to be $\left\{f_{i}(z) \exp [j 2 \pi N(0,1)]\right\}$. Here $N(0,1)$ is a random variable that uniformly distributes in the interval $[0,1)$.

If we have to analyze the effect of $\mathrm{N}$ modes with different azimuthal orders, we can find $\mathrm{N}$-dimensional orthogonal basis from $\left\{f_{i}(z)\right\}$ to approximate the effect of inhomogeneity, according to the representation theorem in strictly normed linear space[13]. The weights for each orthogonal basis can be obtained by matrix transform.

Surface roughness effect caused by $\Delta r(\phi)$ and $\Delta r^{\prime}(\phi)$ can be represented as an elementary function of $\Delta r(\phi)$ and $\Delta r^{\prime}(\phi)$, so we can easily obtain [12]:

$$
\begin{aligned}
& \left\langle F\left\{\left[f_{k}(x)\right]^{i}\right\}\right\rangle=F\left\{\left\langle\left[f_{k}(x)\right]^{i}\right\rangle\right\} \\
& \left\langle F\left\{\left[f_{k}{ }^{\prime}(x)\right]^{i}\right\}\right\rangle=F\left\{\left\langle\left[f_{k}{ }^{\prime}(x)\right]^{i}\right\rangle\right\}
\end{aligned}
$$

Thus, if $\int_{0}^{2 l_{0}}\left|f_{k}(x)\right| d x$ keeps static when the value of $l_{0}$ changes, the value of $\left\langle\left[f_{k}(x)\right]^{i}\right\rangle$ will be the same, which means the $\Delta r(\varphi)$ induced roughness effect will not change. Similarly by keeping $\int_{0}^{2 l_{0}}\left|f_{k}{ }^{\prime}(x)\right| d x$ static, we can keep the effect of $\Delta \mathrm{r}^{\prime}(\varphi)$ unchanged even though the value of $l_{0}$ changes. In this way, we can analyze the effect of $\Delta r(\varphi)$ and $\Delta \mathrm{r}^{\prime}(\varphi)$ respectively.

\section{Simulation and Result Analysis}

To confirm the theory above, we analysis the surface roughness effect on Q-factor of micro-disk as an example. Because we mainly concentrate on the power from the in/out port, the standard operator norm defined in [14] is carefully applied to estimate the transmittance of microdisk. The operator norm is defined as:

$$
\left\langle\|E\|^{2}\right\rangle=\sup _{x \in S, x \neq 0} \frac{\langle(E x, E x)\rangle}{(x, x)}
$$

According to [6], the height of inhomogeneity $\Delta r(\varphi)$ induced scattering for $2 \mathrm{D}$ micro-disk resonator can be written as

$$
\left\langle\left\|E^{(h)}\right\|^{2}\right\rangle \rightarrow \frac{(K \sigma)^{2}}{\left(1+\Lambda^{2}\right)^{2}}
$$

and if the surface is rather smooth, which means $\Lambda$ is fairly small, it is the height of inhomogeneity $\Delta r(\varphi)$, rather than its first derivative $\Delta r^{\prime}(\varphi)$, that is the dominant factor that leads to scattering loss.

To compare the deviation above with the orthogonal basis method we proposed in Section 2, we conduct FDTD simulations with on Ge whisper gallery mode (WGM) micro-disk resonator. The simulation tool is FDTD solution release 8.0. Micro-resonators based on Ge are more and more popular for the merit of especially high Q-factor and graft ability on Silicon based device [11]. It's of great value to have a thoroughly research on the surface roughness effect of Ge micro-disk.

In our simulation, the diameter of micro-disk is $3 \mu \mathrm{m}$. The waveguide coupled along the disk is made of $\mathrm{SiO} 2$ with $0.5 \mu \mathrm{m}$ width at $200 \mathrm{~nm}$ distance from the disk. $\mathrm{N}=$ 1.42 is used as waveguide's reflective index and a value of $\mathrm{n}=4.2$ for Ge micro disk. Surface roughness is manipulated by orthogonal basis $\left\{f_{i}(x)\right\}$ and details of orthogonal basis are carefully calculated following the method described in Section 2. When we investigate the propagation of fundament TE/TM mode, the mode number $\mathrm{n}$ in (9) is set to 1 . Mean variance of roughness $\sigma^{2}$ is $1 \mathrm{~nm}$ and correlation length $L_{c}$ is $50 \mathrm{~nm}$. According to (8-9), if $\phi=\pi / 4$, we can obtain

$$
\begin{aligned}
& L=16 \pi R /(1+8 p) \\
& h \cdot \frac{\sin ^{2}\left[0.01895(1+8 p) l_{0}\right]}{(1+8 p)^{2} / 52.8}\left|1-\exp \left[j 0.0379(1+8 p) l_{0}\right]\right| \\
& =0.4107
\end{aligned}
$$

It's easy to observe from (13) that the larger $p$ is, the more accurate we can calculate because we need to fix the value of $L$ to make $2 \pi R / L$ to be an integer. In our simulation, $p$ is set to be 4 , and the relationship between $h, l_{0}$ indicated in (17) can be further simplified.

As it is stated above, by keeping $\int_{0}^{2 l_{0}}\left|f_{k}(x)\right| d x$ static, according to (10), the scattering loss due to $\Delta \mathrm{r}^{\prime}(\varphi)$ changes with $l_{0}$ increasing from $150 \mathrm{~nm}$ to $400 \mathrm{~nm}$ while the effect of $\Delta r(\varphi)$ will be the same. Similarly, if the value of $\int_{0}^{2 l_{0}}\left|f_{k}{ }^{\prime}(x)\right| d x \quad$ s static when $l_{0}$ s varied, the scattering loss due to $\Delta r(\varphi)$ will change accordingly and the effect of $\Delta \mathrm{r}^{\prime}(\varphi)$ keeps static.

Figure 3 shows the electromagnetic (EM) field distribution when the effect of $\Delta r(\varphi)$ varies with the increase of $l_{0}$. Obviously, Q-factor of micro-disk resonator decrease dramatically as the height of inhomogeneity increases. On the contrary, if the value of $\int_{0}^{2 l_{0}}\left|f_{k}(x)\right| d x$ is a constant, the Q-factor almost keeps unchanged with the alternative of $l_{0}$, as depicted in Figure 4. Figure 5 reveals different sensitivity of micro-resonator's Q-factor on the two scattering mechanism. The effect of $\Delta r(\varphi)$ 


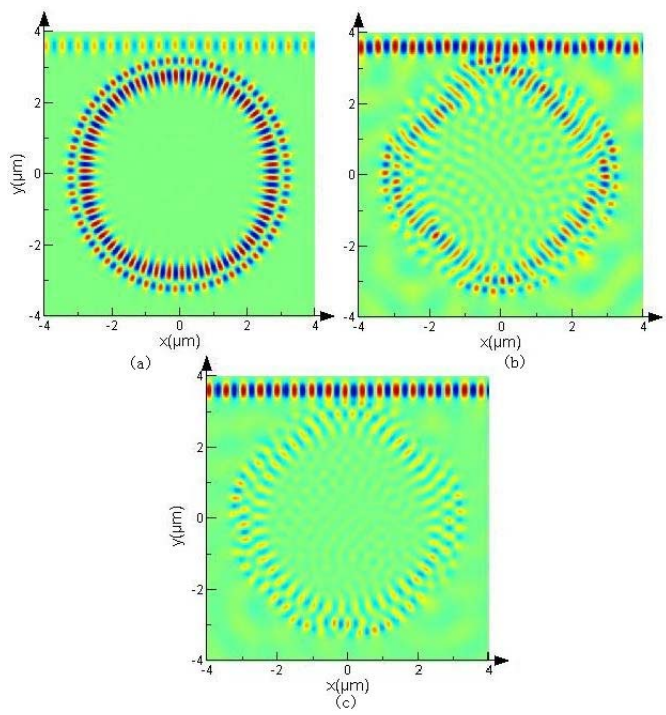

Figure 3. Electromagnetic (EM) field distribution profile at the wavelength of $1548.39 \mathrm{~nm}$ (a) flat surface (b) $I_{0}$ is $200 \mathrm{~nm}$ (c) $l_{0}$ is $400 \mathrm{~nm}$.

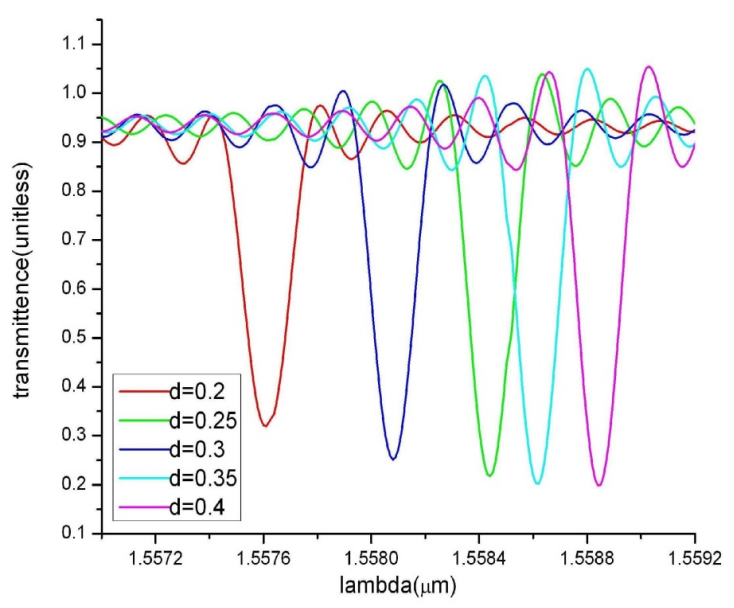

Figure 4. Transmission characteristics as a function of wavelength.

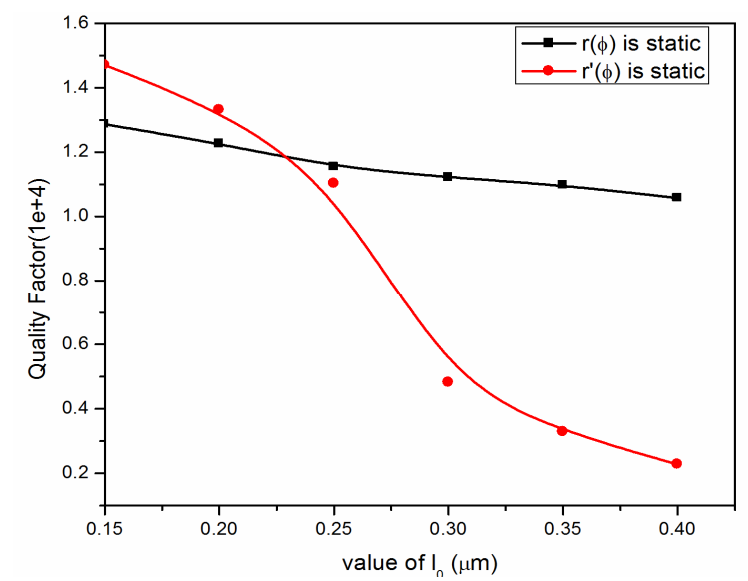

Figure 5. Q-factor as a function of the value of $l_{0}$ in different scattering mechanism. is the dominator factor that leads to the degrading of micro-disk resonator's Q-factor, when side wall roughness is not fairly obvious, i.e. $\sigma \ll L_{c}$. Results of the simulation based on our method fit well with the analysis in [6].

\section{Conclusions}

In this paper, we proposed a novel method base on orthogonal decomposition to analyze the surface roughness effect of micro-resonator. It becomes possible to calculate the scattering loss of $\Delta r(\varphi)$ and $\operatorname{sr}^{\prime}(\varphi)$ respectively through our work, and so we can have a better understanding of the inhomogeneity's effect on micro resonator. Our theory is proved by Fourier Transform and matrix analysis. Finally, we investigated the surface roughness effect on Q-factor of micro-disk resonator, and a FDTD simulation based on orthogonal basis is conducted to validate our deviation.

\section{REFERENCES}

[1] R. Soref, "The past, present, and future of silicon photonics," IEEE Journal of Selected Topics in Quantum Electronics, Vol.12, No.6, 2006, pp.1678- 1687. doi:10.1109/JSTQE.2006.883151

[2] M. Soltani, S. Yegnanarayanan and A. Adibi, "Ultra-High Q Planar Silicon Microdisk Resonators for Chip-Scale Silicon Photonics," Optics Express, Vol.15, No.8, 2007, pp.4694-4704. doi:10.1364/OE.15.004694

[3] L.Q. Ren, X. Wu, M. Li, X. Zhang, L. Liu, and L. Xu, "Ultrasensitive Label-Free Coupled Optofluidic Ring Laser Sensor," Optics Letters, Vol.37, No.18, 2012, pp.3873-3875. doi:10.1364/OL.37.003873

[4] Y. Hu, X. Xiao, H. Xu, X. Li, K. Xiong, Z. Li, T. Chu, Y. $\mathrm{Yu}$, and $\mathrm{J} . \mathrm{Yu}$, “High-speed silicon modulator based on cascaded microring resonators," Optics EXpress,Vol. 20,No.14, 2012, pp.15079-15085. doi:10.1364/OE.20.015079

[5] Q. Li, A. A. Eftekhar, "Azimuthal-Order Variations of Surface-Roughness-Induced Mode Splitting and Scatteringloss in High-Q Microdisk Resonators," Optics Letters, Vol. 37, No.9, 2012, pp. 1586-1588. doi:10.1364/OL.37.001586

[6] E. Ganapolskii and Z. Eremenko, "Effect of Random Surface Inhomogeneities on Spectral Properties of Dielectric-Disk Microresonators: Theory and Modeling at Millimeter Wave Range," Physical Review E, Vol.79, No.4, 2009, p. 041136. doi:10.1103/PhysRevE.79.041136

[7] M. Borselli, T. J. Johnson and O. Painter, "Beyond the Rayleigh Scattering Limit in High-Q Silicon Microdisks: Theory and Experiment," Optics Express, Vol.13, No.5, 2005, p. 1515 . doi:10.1364/OPEX.13.001515

[8] X. Yi and Y. Xiao, "Multiple-Rayleigh-Scatterer Induced 
Mode Splitting in A High-Q Whispering-Gallery-Mode Microresonator," Physical Review A, Vol.83, No.2, 2011, p. 023803 . doi:10.1103/PhysRevA.83.023803

[9] S. Cho and S. Koo, "Surface Roughness Effect on Q-Factor of Ge Whispering Gallery Mode Microdisk Resonator," Optical Society of America, 2011.

[10] N. M. Makarov and Y. V. Tarasov, "Electron Localization in Narrow Surface-Corrugated Conducting Channels: Manifestation of Competing Scattering Mechanisms," Physical Review B, Vol. 64, No.23, 2001, p. 235306. doi:10.1103/PhysRevB.64.235306
[11] H.-Y. Yu, M. Ishibashi, J. -H. Park, M. Kobayashi, and K. C. Saraswat, "p-Channel Ge MOSFET by Selectively Heteroepitaxially Grown Geon $\mathrm{Si}$, ' IEEE Electron Device Letters, Vol. 30, No. 6, 2009, pp. 675-677. doi:10.1109/LED.2009.2019847

[12] W. A. Gardener, "Introduction to Random Processes: With Applications to Signals and Systems," New York, NY: McGraw-Hill 1990

[13] R. A. Horn and C. R. Johnson, "Matrix Analysis," Cambridge University Press, Cambridge, 1990.

[14] E. Kreyszig, "Introductory functional analysis with applications," John Wiley \& Sons, New York, 1989. 